\title{
miR-222 regulates sorafenib resistance and enhance tumorigenicity in hepatocellular carcinoma
}

\author{
KAI LIU, SONGYANG LIU, WEI ZHANG，BAI JI，YINGCHAO WANG and YAHUI LIU \\ Department of Hepatopancreatobiliary Surgery, The First Hospital, \\ Jilin University, Changchun, Jilin Province, P.R. China
}

Received June 11, 2014; Accepted July 24, 2014

DOI: $10.3892 /$ ijo.2014.2577

\begin{abstract}
The miR-222 cluster has been demonstrated to function as oncomiR in human hepatocellular carcinoma (HCC). miR-222 confers chemotherapy drug resistance in various cancers, including HCC. However, the effects and mechanisms by which miR-222 regulates liver tumorigenicity and confers sorafenib (SOR) resistance remain unclear. Here we first investigated the miR-222 effect on proliferation, cell cycle, apoptosis, migration and invasion of HCC. Our results demonstrated that miRNA inhibitors specially targeting miR-222 significantly suppressed cellular proliferation, migration, invasion and G1/S transition of the cell cycle, and induced cell apoptosis in HepG2 cells. In addition, we investigated whether miR-222 confers SOR resistance in HepG2 cells to explore it roles in acquisition of drug resistance. The results showed that miR-222 inhibitors induced sensitivity to the antitumor effect of sorafenib in human HepG2 cells. Importantly, our study also showed that miR-222 could regulate the expression of phosphorylation PI3K and AKT, which might contribute to miR-222 conferred SOR resistance in HepG2 cells. In conclusion, this study demonstrates that miR-222 can promote cell proliferation, migration and invasion, and decrease cell apoptosis, as well as enhance the resistance of HCC cells to sorafenib miR-222 through activating the PI3K/AKT signaling pathway.
\end{abstract}

\section{Introduction}

Hepatocelluar carcinoma (HCC) is the third leading cause of cancer-related deaths worldwide, and the burden of this devastating cancer is expected to increase further in the coming years (1). More than $90 \%$ of HCC cases develop in chronically inflamed liver as a result of viral hepatitis, alcohol abuse and in increasing incidence in patients with non-alcoholic

Correspondence to: Professor Yahui Liu, Department of Hepatopancreatobiliary Surgery, The First Hospital, Jilin University, Changchun, Jilin Province, P.R. China

E-mail: liuyahui588@126.com

Key words: miR-222, miRNA, sorafenib, hepatocellular carcinoma fatty liver disease $(2,3)$. The overall poor survival of $\mathrm{HCC}$ patients is primarily attributed to the late disease presentation, which rules out curative surgery for the majority of patients at intermediate or advanced stages (4). Chemotherapy is an important component in the treatment of patients with late HCC. However, drug-resistance minimizes the effectiveness of such therapy in a large number of patients (5-8). Drug resistance is a complex phenomenon, with multiple factors and mechanisms contributing to the resistance (5-8). Although advances in the fields of resistance-associated proteins (drug transporters, metabolic enzymes and target molecules) and DNA repair or cell apoptosis pathways, to date, there is no validated drug-response/resistant biomarker available in clinical settings, and the underlying mechanisms of acquisition of resistance to chemotherapeutic agents remain poorly understood. Thus, the identification of predictive molecular factors for tumor recurrence and understanding the roles of these markers in the molecular genetic mechanisms underlying HCC tumor recurrence would result in improved overall clinical management of patients with HCC.

The role of microRNAs (miRNAs, miRs) in regulating drug resistance has been reported. MicroRNAs (miRNA) belong to a class of endogenously expressed small non-coding RNAs with 19-25 nucleotides in length that regulate gene expression by either directing messenger RNA (mRNA) degradation or repressing posttranscriptional protein translation through binding to the $3^{\prime}$ untranslated region (UTR) of targeted gene transcript (9). Increasing evidence has demonstrated that miRNAs can mediate gene expression in a broad spectrum of regulatory pathways and are therefore believed to play essential roles in a range of biological processes, such as embryonic development, cell proliferation, differentiation, migration, apoptosis, and signal transduction (10). Currently, more than 2,000 mature miRNA molecules have been identified or predicted in human-origin cells and tissues (The miRBase Sequence Database-Release 19.0), with an estimated $30 \%$ of human genes targeted (10). Despite the precise function of many of the predicted $\sim 800$ human miRNAs many are still undefined, several cellular miRNAs have already been established as crucial regulators of cell growth, differentiation, and apoptosis by their control of critical tumor suppressors and oncogenes, such as the RAS by Let-7, BCL 2 by miR-15a and miR-16-1, PTEN by miR- 21, and E2F1 by miR-17-miR-92, as well as PTEN by miR-221/222 (11-15). Accumulating evidence 
suggests that miRNAs are associated with every aspect of cancer biology, including acquisition of resistance to various chemotherapeutic agents (16).

Accumulating evidence has demonstrated that microRNA-222 (miR-222) plays a crucial role in cell growth, oncogenesis, invasion, migration and drug resistance in tumor cells $(17,18)$, and overexpression of miR-222 has been found in several types of cancers, such as breast cancer, colorectal carcinoma, glioblastoma, colorectal carcinoma, as well as liver cancer (19-23). In particular, several studies demonstrated that miR-222 is involved in resistance to several chemotherapeutic drugs. For example, Miller et al found that the miR-221/222 confers tamoxifen resistance in breast cancer (20). Zhong et al demonstrated that miR-222 was involved in adriamycin (Adr) and docetaxel (Doc) resistance via targeting PTEN (24). Garofalo and his collaborators showed that miR-221/222, by targeting PTEN and TIMP3 tumor suppressors, induce TRAIL resistance and enhance cellular migration (25). Nevertheless, the possible roles of miR-222, and whether it increases resistance of sorafenib in hepatocellular carcinoma remain unclear. In the present study, the miR-222 on the carcinogenesis of HCC and the underlying mechanisms were examined. In addition, we investigated the role of miR-222 alterations in acquiring drug-resistance and identified miR-222 that could change the drug-resistance sorafenib of HCC cells in vitro.

\section{Materials and method}

Study population. This study retrospectively enrolled patients who had undergone liver resections for primary HCC between July 2009 and July 2012 that were identified from a search of the archival surgical pathology files of the Department of Thoracic Surgery, The First Hospital, Jilin University, Changchun, Jilin Province, China. None of the patients had received chemotherapy or radiotherapy before surgery. During the surgical procedure, samples of malignant liver tissue and samples from adjacent non-cancer tissue $(>5 \mathrm{~cm}$ away from the tumor site, cirrhosis tissue was excluded) were taken. All specimens were frozen and stored at $-80^{\circ} \mathrm{C}$ until used.

The diagnosis of HCC was confirmed histopathologically (26). Data on all subjects were obtained from medical records, pathology reports and personal interviews with the subjects. The data collected include age, gender, overall survival and HCC features such as tumor number, size and growth phase. Clinical stage of HCC was evaluated on the basis of the TNM classification system (27). The Child-Pugh score allowed to categorize HCC patients in Child-Pugh grades A, B and C (28).

All patients gave written informed consent to participate in the study. This study was approved by the Ethics Committee of Jilin University, Changchun, Jilin Province, China.

Cell lines and cell culture. The human HCC cell lines HepG2 and the normal human hepatocyte cell line HL-7702 was purchased from the American Type Culture Collection (ATCC, VA, USA). HepG2 and HL-7702 were cultured in Dulbecco's modified Eagle's medium (DMEM) (Sigma-Aldrich, St. Louis, MO, USA) supplemented with $1 \%$ penicillin/streptomycin (100 mg/l, Gibco-BRL, Grand Island, NY, USA) and 10\% heat-inactivated fetal calf serum (FCS) (Invitrogen, Carlsbad, CA, USA).

Cell transfection. miR-222 mimics and miR-222 inhibitors and corresponding negative controls were purchased from Ambion, Life Technologies (Austin, TX, USA). The Oligofectamine $^{\mathrm{TM}}$ transfection reagent from Invitrogen, Life Technologies was used for cell transfection according to the manufacturer's instructions. Final concentration for miRNA mimics was $30 \mathrm{nM}$, for miRNA inhibitor was $50 \mathrm{nM}$.

miRNA real-time RT-PCR analysis. Total RNA of cell or tissue, including miRNAs, were extracted using the Qiagen miRNeasy Mini kit (catalogue no. 217004; Qiagen, Hilden, Germany) according to the manufacturer's instructions. The purity and concentration of RNA were determined by using a dual-beam ultraviolet spectrophotometer (Eppendorf, Hamburg, Germany). Then, the RNA was reversely transcribed into cDNA using the Universal cDNA synthesis kit from Exiqon (Woburn, MA, USA) following the manufacturer's instructions. For qPCR, we utilized a miRCURY LNA ${ }^{\mathrm{TM}}$ Universal RT microRNA PCR system (Exiqon) to quantify the mature miRNA expression levels on an ABI 7900 HT Sequence Detection System (Applied Biosystem). For detection of miR-222, and U6 snRNA expression, specific primers were obtained from Exiqon, i.e., U6 snRNA PCR primer set (product no. 203907) and LNA ${ }^{\mathrm{TM}}$ hsa-miR-222 PCR primer set (product no. 204551). qPCR was performed using the Universal RT SYBR ${ }^{\circledR}$ Green master mix from Exiqon (product no. 203450) according to the manufacturer's instructions. The qPCR conditions were: an initial $95^{\circ} \mathrm{C}$ for $5 \mathrm{~min}$ and followed by 40 cycles of $95^{\circ} \mathrm{C}$ for $10 \mathrm{sec}$ and $60^{\circ} \mathrm{C}$ for $30 \mathrm{sec}$. A dissociation curve was established after each PCR in order to verify amplification specificity. The integrity of the miRNA and the efficiency of qPCR in each sample were confirmed by the endogenous control U6 small RNA. Negative control experiments were set without cDNA template. The relative quantification of each miRNA was presented as the fold change after normalized to the U6 RNA for the equation $2^{-\Delta \Delta \mathrm{Ct}}$ in Rotor-Gene 6000 Series Software 1.7 (Qiagen).

Cell proliferation assay. Cell viability was assessed by CCK-8 assay (Cell Counting Kit-8, Dojindo, Japan) was performed. In brief, $5 \times 10^{3}$ cells/well was seeded in 96-well plates. The proliferative activity was determined at the end of different experimental periods $(24,48,72,96$ and $120 \mathrm{~h})$ using CCK-8 assay according to the manufacturer's instructions. When the media changed from red to yellow, the absorbance value at a wavelength of $450 \mathrm{~nm}$ was detected by an enzyme-linked immunosorbent assay reader (Thermo Labsystems, Finland). The experiment was performed at least three times with similar results.

The proliferation rate of cells was determined by measuring the incorporation of bromodeoxyuridine (BrdU) into the genomic DNA. In brief, $2 \times 10^{3}$ cells/well was seeded in 96-well plates. A 5-bromodeoxyuridine (BrdU) incorporation assay was performed using the BrdU Cell Proliferation Assay kit (Chemicon, Temecula, CA, USA) according to the manufacturer's instructions. Plates were read at a dual wavelength 
of $450 / 550 \mathrm{~nm}$, and the growth rate of cells was calculated as described previously (23).

Cell cycle analysis. The cell cycle distribution was analyzed by using FACScan flow cytometry. In brief, cells were starved in DMEM supplemented with 5\% charcoal-stripped serum or $0.5 \%$ regular FBS. After $24 \mathrm{~h}$, medium was changed to DMEM with $10 \%$ normal FBS. Cells were harvested at different time-points and cell cycle parameters were determined using laser scanning cytometry. Cells were processed by standard methods by using propidium iodide (PI, $20 \mu \mathrm{g} / \mathrm{ml}$; Sigma) staining of cell DNA. Ten thousand cells per sample were analyzed by flow cytometry using a FACS can flow cytometer (BD Biosciences, Mansfield, MA, USA).

Apoptosis analysis. To determine the number of apoptotic cells, TUNEL assay was performed. In brief, cellular DNA fragmentation was measured with the ApoTag Red in situ apoptosis detection kit (Chemicon International, CA, USA) according to the manufacturer's instructions when HepG2 cells were transfected with miR-222 inhibitor and corresponding negative control for $48 \mathrm{~h}$. To quantify the apoptotic cells, the terminal deoxynucleotidyl transferase-mediated nick end-labeling (TUNEL)-positive cells were counted using a confocal microscopy (Olympus, Tokyo, Japan).

In addition, we also detected caspase-3, -8 and -9 activity by ELISA as an additional indicator of apoptosis.

Caspase activity. The activity of caspase- $3,-8$ and -9 was determined by caspases colorimetric protease assay kits (Millipore Corp., Billerica, MA, USA) according to the manufacturer's instructions. In brief, cells were washed twice with ice-cold PBS and harvested by centrifugation $700 \mathrm{~g}$ for $10 \mathrm{~min}$. The cell pellets were then lysed in $150 \mu$ lbuffer provided in the kit. Protein concentrations of lysates were measured by the Lowry method. An aliquot of lysates $(80 \mu 1)$ was incubated with $10 \mu \mathrm{l}$ substrate of each caspase at $37^{\circ} \mathrm{C}$ for $2 \mathrm{~h}$. Samples were analyzed at $405 \mathrm{~nm}$ in a microplate reader (Thermo Fisher Scientific Inc., Waltham, MA, USA). The relative caspase activity of the control group was referred as 100 .

Wound-healing assay. To assess the effect of miR-222 on cell migration, wound-healing assay was performed. In brief, HepG2 cells transfected miR-222 inhibitor and corresponding negative controls, were seeded in $6-\mathrm{cm}$ dish with $1.5 \times 10^{6}$ wells per dish and cultured for $24 \mathrm{~h}$, the linear wound of cellular monolayer was created by scratching confluent cell monolayer using a plastic pipette tip. The monolayer of scratched cell was washed by PBS to remove debris. After incubation at $37^{\circ} \mathrm{C}$ with $5 \% \mathrm{CO}_{2}$ for $48 \mathrm{~h}$, area of migration was photographed under light microscope for evaluation. All experiments were performed in triplicate.

Transwell invasion assay. The invasion capacity of HepG2 cells was performed in vitro using Transwell Chambers (Corning, Tewksbury, MA, USA) in which the two chambers were separated by a Matrigel-coated polycarbonate membrane $(8-\mu \mathrm{m}$ pore size). In brief, HepG2 cells transfected with miR-222 inhibitor and corresponding negative controls, was seeded into Cell Culture Insert ( $8-\mu \mathrm{m}$ pore size; Falcon, BD Bioscience), precoated with $25 \mu \mathrm{l}$ of $20 \%$ Matrigel ( $2-3 \mathrm{mg} / \mathrm{ml}$ protein), and then placed in a 24 -well plate (Falcon) with $1 \times 10^{5}$ wells per well. Cell were fixed and stained with $0.5 \%$ crystal violet after they had been cultured at $37^{\circ} \mathrm{C}$ for $48 \mathrm{~h}$. The cells on the top of the Cell Culture Insert were removed by wiping with a cotton swab, and cell invasion was observed with an immunofluorescence microscope by counting the cells that had invaded into the bottom of the Cell Culture Insert. All experiments were performed in triplicate.

In vitro sorafenib treatment. HepG2 cells were transfected with miR-222 mimic and miR-222 inhibitors and corresponding negative controls as described above, and at $48 \mathrm{~h}$ after transfection, the medium was changed to DMEM containing sorafenib (Bayer HealthCare Pharmaceuticals Inc., USA). After 72 h, cell viability was assessed using a CCK8 assay, and apoptosis was measured by TUNEL. In addition, caspase- 3 and caspase- 8 activity were determined as above described.

Western blot analysis. Whole-cell lysates $(50 \mu \mathrm{g})$ were collected after 48-h transfection of miRNA mimics or inhibitors, and then homogenized in a lysis buffer (Tris- $\mathrm{HCl} 50 \mathrm{mmol} / \mathrm{l}$, EDTA $5 \mathrm{mmol} / \mathrm{l}, \mathrm{NaCl} 150 \mathrm{mmol} / \mathrm{l}$, sodium deoxycholate 1\%, $\mathrm{Na}_{3} \mathrm{VO}_{4} 500 \mu \mathrm{mol} / \mathrm{l}$, Triton X-100 0.5\%, AEBSF $10 \mu \mathrm{mol} / \mathrm{l}$, $\mathrm{NaF} 10 \mathrm{mmol} / \mathrm{l}$ ) on ice for $30 \mathrm{~min}$. Cell lysates were insolated by centrifugation at $10,000 \mathrm{~g}$ for $15 \mathrm{~min}$, and protein concentrations were determined using the Bradford reagent (Sigma, Germany). Equal amounts of protein ( $20 \mu \mathrm{g} /$ lane) from the cell lysates were separated on an 8-15\% SDS-polyacrylamide gel (SDS-PAGE) and transferred onto nitrocellulose membranes (Santa Cruz Biotechnology, Inc., Santa Cruz, CA, USA). The membrane was incubated for $2 \mathrm{~h}$ in PBS plus $0.1 \%$ Tween-20 and 5\% non-fat skim milk to block non-specific binding. Then the membranes were incubated overnight at $4^{\circ} \mathrm{C}$ with primary antibodies. After washing, the membranes were incubated with the appropriate HRP-conjugated secondary antibody (Amersham Pharmacia Biotech, Piscataway, NJ, USA) at room temperature for $1 \mathrm{~h}$ followed by ECL staining. The following antibodies were used for western blotting: anti-cyclin D1, anti-cyclin D3, anti-p27 and anti- $\beta$-actin were purchased from Santa Cruz Biotechnology, Inc. Anti-PI3K and anti-phosphorylated PI3K (p-PI3K; Tyr458); anti-AKT and anti-phosphorylated AKT (p-Akt; S473) were purchased from Cell Signaling Technology (Beverly, MA, USA).

Statistical analysis. All data are expressed as mean \pm standard deviation (SD) Statistical analysis between two samples was performed using Student's t-test. Statistical comparison of more than two groups was performed using one-way ANOVA followed by a Tukey post hoc test. The relationship between miR-222 expression level and clinical and pathological variables was analysed using Pearson's $\chi^{2}$ test. The relationship between miR-222 expression and patient survival was analysed using univariate analysis (Kaplan-Meier). Graphpad Prism 6.0 software (GraphPad Software, San Diego, CA, USA) and SPSS ${ }^{\circledR} 19.0$ (SPSS Inc., Chicago, IL, USA) for Windows ${ }^{\circledR}$ were used for statistical analyses. A value of $\mathrm{P}<0.05$ was taken as an indication of statistical significance. All the figures shown in this report were obtained from at least three independent experiments with similar results. 
A

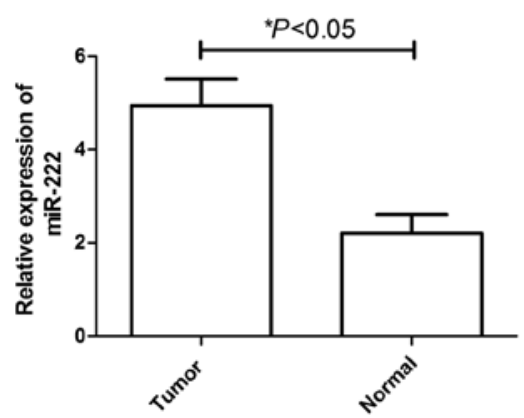

B

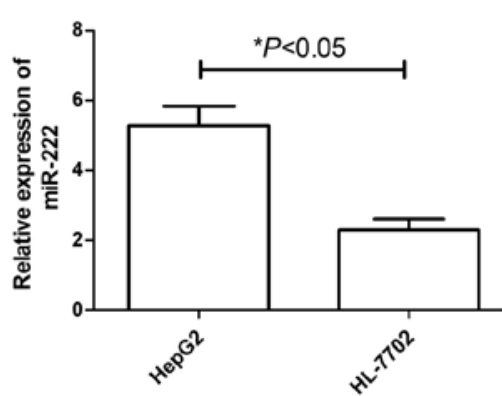

C

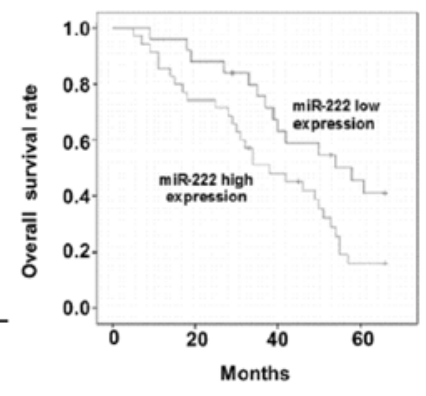

Figure 1. miR-222 was upregulated in HCC tissues and HCC cell lines, and was associated with survival. (A) The relative expression levels of miR-222 in 90 paired human HCC tissues were measured by real-time quantitative RT-PCR (qRT-PCR), ${ }^{*} \mathrm{P}<0.05$ versus adjacent non-tumor tissues. (B) The relative expression levels of miR-222 were measured by qRT-PCR in one normal human hepatocyte cells and one HCC cell line (HepG2), $\mathrm{P}<0.05$ versus normal hepatocyte cells (HL-7702). (C) Kaplan-Meier analyses of overall survival in 90 HCC patients according to the expression levels of miR-222.

\section{Results}

miR-222 was upregulated in HCC tissues and HCC cell lines. In the present study, we detected miR-222 expression levels in tumor tissues and adjacent non-tumor tissues from 90 patients with HCC and in the HCC cell lines. As revealed by real-time quantitative RT-PCR (qRT-PCR) analysis, the expression level of miR-222 was significantly upregulated in tumor tissues compared to expression in the matched adjacent non-tumor tissues (Fig. $1 \mathrm{~A}, \mathrm{P}<0.05$ ) As observed in HCC tissues, the miR-34a expression level was substantially upregulated according to qRT-PCR analysis in HepG2 cells compared to HL-7702 cells (the normal human hepatocyte cell line) (Fig. 1B, $\mathrm{P}<0.05$ ).

In addition, we compared the clinicopathological factors of the high and low miR-34a expression groups (Table I) and found that there was no correlation between the miR-222 expression levels and age, sex and Child-Pugh grade, but the relative miR-222 expression levels were significantly positively correlated with TNM stage and the presence lymph node metastasis, tumor size, differentiated ( $\mathrm{P}<0.05$ for both comparisons). The relative miR-222 expression level was significantly higher in patients with stage IIII-V HCC compared with patients with stage I-II disease $(\mathrm{P}<0.05)$; and in patients with lymph node involvement compared with patients without lymph node involvement $(\mathrm{P}<0.05)$; and in patients with lager tumor size $(>5 \mathrm{~cm})$ compared with small tumor size $(<5 \mathrm{~cm})$.

As tumor grade and stage significantly affect HCC treatment and outcome, the observed dysregulation of miR-222 may be linked to HCC survival. A Kaplan-Meier survival analysis indicated that high miR-222 expression correlated with shorter overall survival (Fig. 1C, $\mathrm{P}=0.002$ ). Thus, the miR-222 expression status in tumors can predict HCC survival.

Effects of miR-222 on cell proliferation in HepG2 cells. In view of the high expression of miR-222 in HepG2 cells, we examined the effect of miR-222 on cell proliferation of HepG2. miR-222 mimics and miR-222 inhibitors were transfected into HepG2 cells, respectively, followed by CCK-8 assays and BrdU incorporation assays. As shown in Fig. 2A, the viability of HepG2 cells was markedly increased by transfection miR-222 mimics ( $\mathrm{P}<0.05$ compared to control), and
Table I. Correlations between the relative level of miR-222 in tumor tissue of patients with HCC and clinicopathological features of HCC.

\begin{tabular}{llcc}
\hline Feature & n & $\begin{array}{c}\text { Relative miR-222 } \\
\text { level }\left(2^{-\Delta \Lambda C T}\right)^{\mathrm{a}}\end{array}$ & $\begin{array}{c}\text { Statistical } \\
\text { significance }^{\mathrm{b}}\end{array}$ \\
\hline
\end{tabular}

Age, years

$\begin{array}{llll}<55 & 48 & 4.891 \pm 0.678 & \text { NS } \\ >55 & 42 & 4.762 \pm 0.597 & \end{array}$

$\geq 55$

$4.762 \pm 0.597$

Gender

$\begin{array}{llll}\text { Male } & 52 & 4.793 \pm 0.682 & \text { NS } \\ \text { Female } & 48 & 4.912 \pm 0.792 & \end{array}$

Clinical stage

$\begin{array}{llll}\text { I-II } & 54 & 3.934 \pm 0.589 & \mathrm{P}<0.05 \\ \text { III-IV } & 36 & 5.441 \pm 0.822 & \end{array}$

Tumor size, $\mathrm{cm}$

$\begin{array}{llll}<5 & 60 & 4.123 \pm 0.778 & \mathrm{P}<0.05 \\ \geq 5 & 30 & 5.421 \pm 0.898 & \end{array}$

Childs classification

$\begin{array}{lrrr}\text { A } & 55 & 4.523 \pm 0.654 & \text { NS } \\ \text { B } & 18 & 4.674 \pm 0.711 & \\ \text { C } & 7 & 5.123 \pm 0.813 & \end{array}$

Regional lymph

node involvement

$\begin{array}{llll}\text { No } & 61 & 4.174 \pm 0.784 & \mathrm{P}<0.05 \\ \text { Yes } & 39 & 5.842 \pm 0.924 & \end{array}$

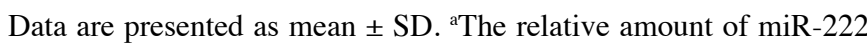
was calculated using the $2^{-\Delta \Delta C \mathrm{CT}}$ method where $\Delta \Delta C=\left(C_{\mathrm{T} \text { miR-222- }} C_{\mathrm{T} \mathrm{U} 6}\right)$ of the HCC group - $\left(C_{\mathrm{T} \text { miR-222 }} C_{\mathrm{T} \mathrm{U} 6}\right)$ of the control group. ${ }^{\mathrm{b}}$ Differences in the miR-222 levels were analysed using Student's t-test. NS, no statistically significant difference between groups $(\mathrm{P} \geq 0.05)$.

the enhanced effect of miR-222 mimics on cell proliferation can be observed beginning on day 2 ; it became more obvious on days 4 and 5 ( $\mathrm{P}<0.05$, Fig. 2A). On the other hand, miR-222 inhibitors significantly inhibited cell proliferation compared to 
A

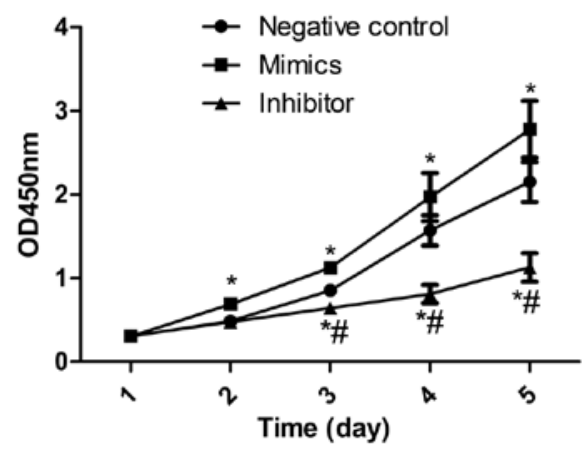

B

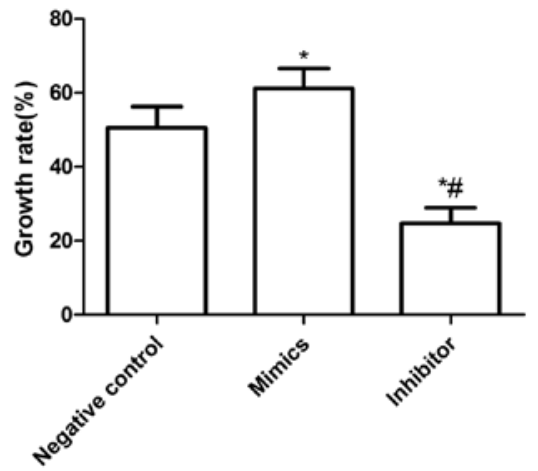

Figure 2. miR-222 affected cell proliferation in HepG2 cells. (A) miR-222 overexpression increased cell viability as detected by CCK8 assay in HepG2 cells. Cells transfected with the miR-222 mimic, miR-222 inhibitor and corresponding negative control for 3 days were seeded in 96-well plates, and cell viability was determined at indicated times. (B) miR-222 overexpression increased cell viability as detected by a BrdU incorporation assay. The growth rate of HepG2 was calculated. All assays were performed in triplicate. " $\mathrm{P}<0.05$ versus the negative control; ${ }^{~} \mathrm{P}<0.05$ versus mimics.

A
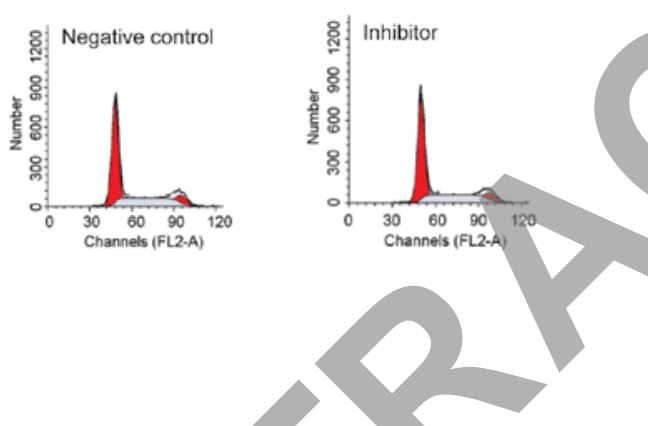

C

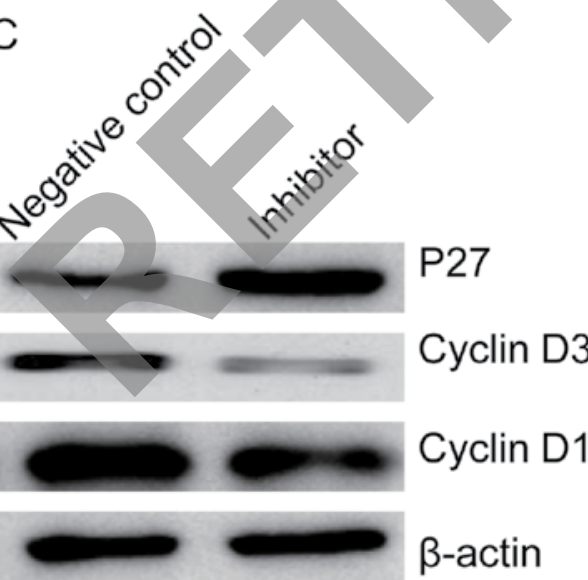

B

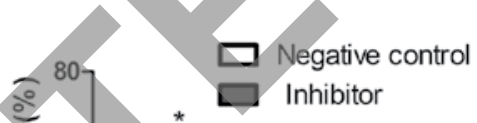

D
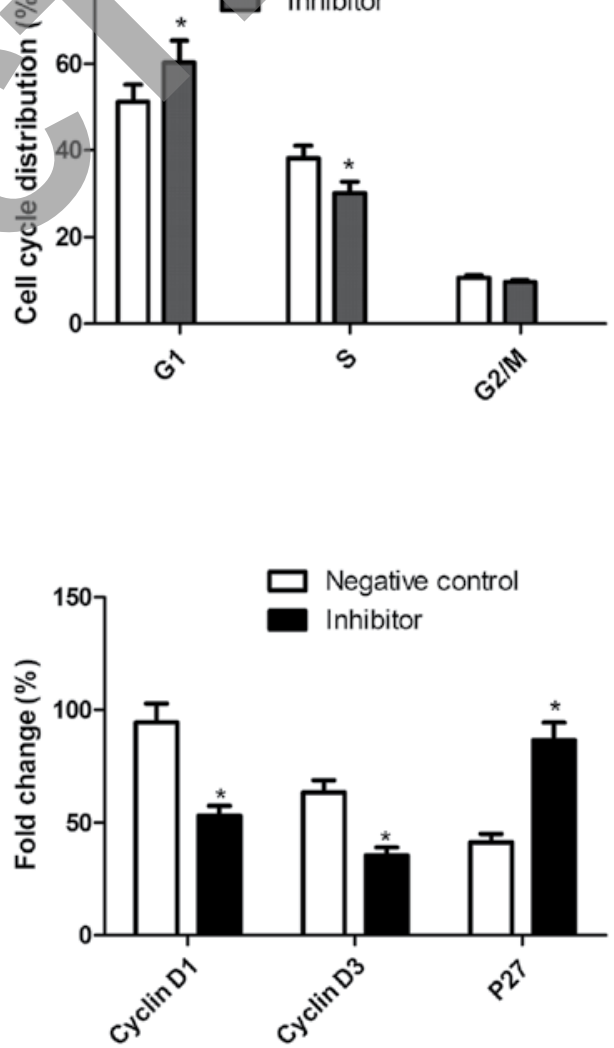

Figure 3. Effects of miR-222 inhibitor on HepG2 cell cycle distribution. (A) Cell DNA content distribution in each phase. (B) Percentage of cells distributed in each phase of the cell cycle. (C) Western blot analysis of cyclin D1, cyclin D3 and P27 protein expression after transfected with miR-222 inhibitor and corresponding negative control. $\beta$-actin was used to an internal control. (D) Relative quantification of cyclin D1, cyclin D3 and P27 protein by densitometric analysis; ${ }^{*} \mathrm{P}<0.05$ versus the negative control.

control group at different times $(\mathrm{P}<0.05$, Fig. 2A). Consistent with the CCK8 assays, BrdU incorporation assays also demonstrated that the proliferation rate of the miR-222 mimic group was significantly increased compared to control group and miR-222 inhibitor group $(\mathrm{P}<0.05$, Fig. $2 \mathrm{~B})$. These findings suggest that the miR-222 greatly increased cell proliferative ability in HepG2 cells.
Effects of the miR-222 inhibitor on cell cycle in HepG2 cells. In order to determine the effects of miR-222 on the cell cycle, FACScan flow cytometry was performed. A flow cytometry analysis revealed that G1-phase cell population was observed in the cells transfection miR-222 inhibitor as compared with the cells transfection negative control ( $\mathrm{P}<0.05$, Fig. $3 \mathrm{~A}$ and $\mathrm{B})$. In addition, transfection with miR-222 inhibitor resulted in 
A
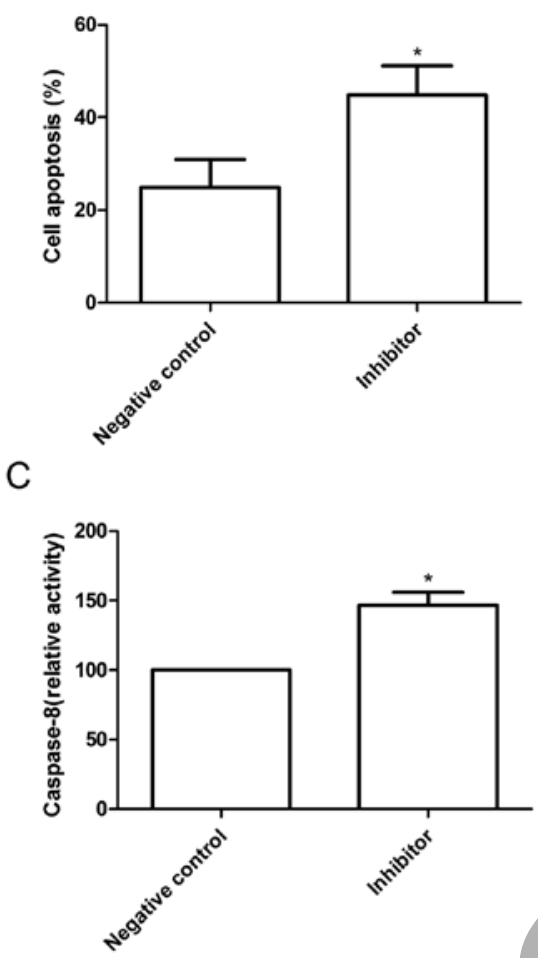

B

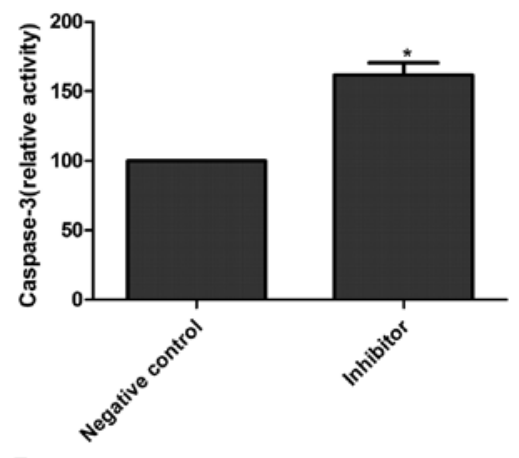

D

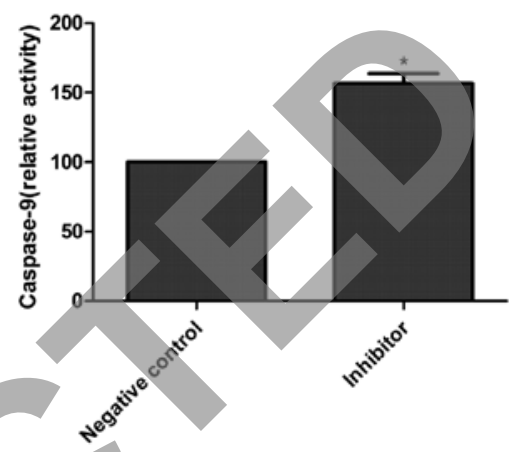

Figure 4. Effects of miR-222 inhibitor on HepG2 cell apoptosis. (A) Cell apoptosis was determined by TUNEL after cells transfected with miR-222 inhibitor and corresponding negative control. Caspase-3 (B), caspase-8 (C) and caspase-9 (D) activity were determined by ELISA, ${ }^{*} \mathrm{P}<0.05$ versus negative control.

a much lower percentage of cells in $\mathrm{S}$ phase compared with those transfected with negative control $(\mathrm{P}<0.05$, Fig. $3 \mathrm{~A}$ and $\mathrm{B})$. There were no significant differences in cells in $\mathrm{G} 2 / \mathrm{M}$ phases among the groups.

Next, we analyzed the effects of miR-222 inhibitor on the expression of cell cycle relevant proteins, such as cyclin D1, cyclin D3 and P27. As shown in Fig. 3C and D, compared to cells transfected with negative control, p27 expression was dramatically increased, whereas, cyclin D1 or cyclin D3 expression significantly decreased in cells transfected with miR-222 inhibitor ( $\mathrm{P}<0.05$, Fig. 3D). These results suggested that miR-222 may regulate the cell cycle.

Effects of miR-222 inhibitor on cell apoptosis in HepG2 cells. In order to further investigate the effect of miR-222 on cell apoptosis in HepG2 cells, TUNEL assays were performed. It was found that cells transfected with miR-222 inhibitor could significantly induce cell apoptosis compared to cells transfected with negative control $(\mathrm{P}<0.05$, Fig. $4 \mathrm{~A})$.

Next, we analyzed the effects of miR-222 on caspase- $3,-8$ and -9 activity. As shown in Fig. 4B-D, caspase-3, -8 and -9 activity in cells transfected with miR-222 inhibitor showed significantly increased compared with those cells transfected with negative control $(\mathrm{P}<0.05)$. These results suggest that miR-222 inhibitor can induce cell apoptosis in HepG2 cells.

Effects of miR-222 inhibitor on cell migration and invasion in HepG2 cells. To ascertain the inhibitory effect of miR-222 inhibitor on cell motility in vitro, wound-healing assay was performed to investigate their effects on the migration poten- tial of HepG2 cells. A scratch was introduced into confluent monolayers cells transfeced with miR-222 inhibitor and corresponding negative control, and the time-dependent movement of cells into the injured area was monitored microscopically. Cells began migrating $6 \mathrm{~h}$ after scratching. After $24 \mathrm{~h}$, cells transfected with miR-222 inhibitor migrated significantly less than those in the cells transfected with negative control $(\mathrm{P}<0.05$; Fig. 5A and $\mathrm{B})$.

Next, the ability of miR-222 inhibitor to reduce the invasiveness of HepG2 cells was further investigated using the transwell system assay. It was found that invasion was also significantly decreased in transfected with miR-222 inhibitor compared to cells transfected with negative control $(\mathrm{P}<0.05$; Fig. 5C and D).

Effects of miR-222 inhibitor and sorafenib on the HepG2 cells. Sorafenib is the only oral multi-kinase inhibitor recently approved by the FDA with demonstrated efficacy in enhancing the overall survival of advanced HCC. It is known that some microRNAs can improve the resistance of cancer cells to chemotherapeutic agents, therefore we tested whether miR-222 could increase the effect of sorafenib on HCC cells. For this purpose, we first evaluated the effect of expression of miR-222 with sorafenib on cellular proliferation. As shown in Fig. 6A, sorafenib treatment decreased the viability of HepG2 cells, as determined by CCK8 assay, however, HepG2 cells by transfection with miR-222 mimic were more resistance to sorafenib treatment than cells transfected with negative control $(\mathrm{P}<0.05)$, whereas, cells transfected with the miR-222 inhibitor were more susceptible to sorafenib treatment than the cells transfected with negative control $(\mathrm{P}<0.05)$. 
A

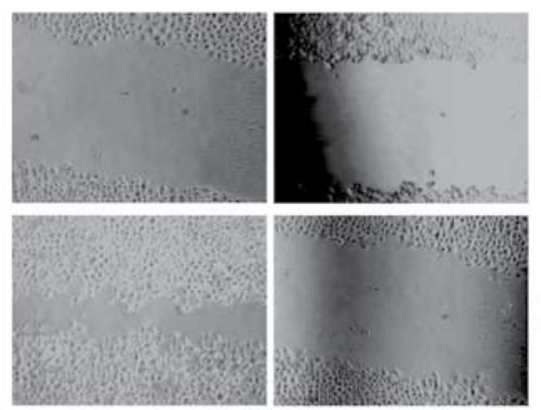

Negative control Inhibitor

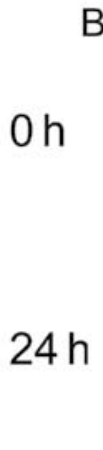

B

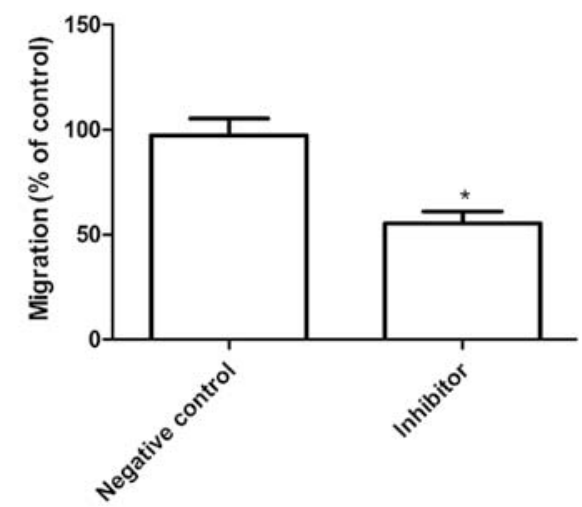

C
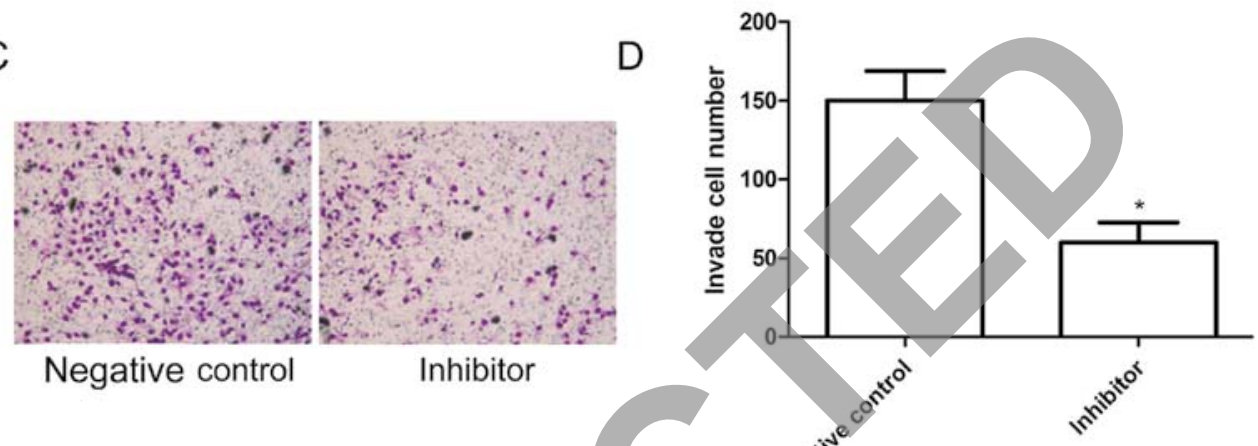

Figure 5. Effects of miR-222 inhibitor on HepG2 cell migration and invasion. (A) Cell migration was determined by wound-healing assay after cell transfection with miR-222 inhibitor and corresponding negative control. Micrographs of cells were taken at 0 and $24 \mathrm{~h}$ after monolayer wounding. (B) Migration number was counted. (C) Cell invasion was determined by Matrigel after transfected with miR-222 inhibitor and corresponding negative control. (D) Histogram of invaded cells of each group, normalized to the control cells after transfection with miR-222 inhibitor and corresponding negative control; ${ }^{*} \mathrm{P}<0.05$ versus negative control.

A

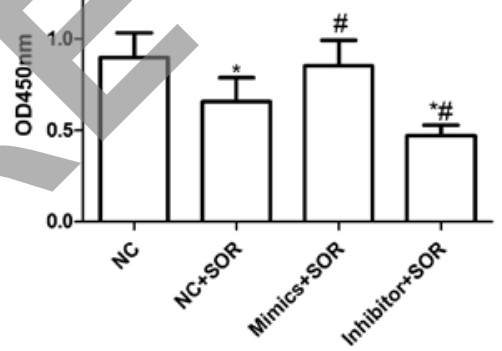

C

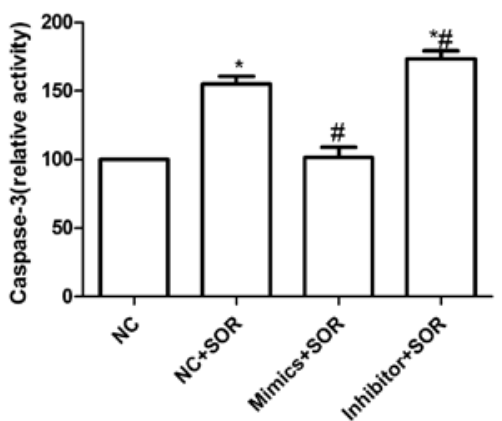

B

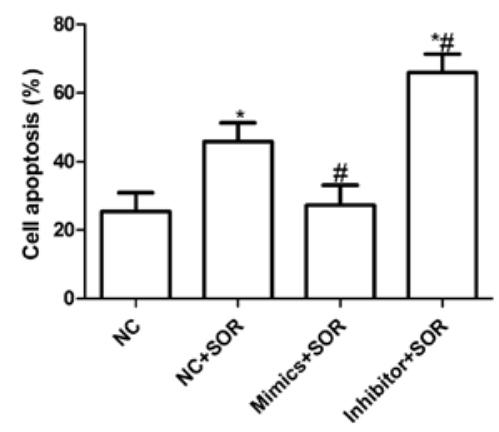

D

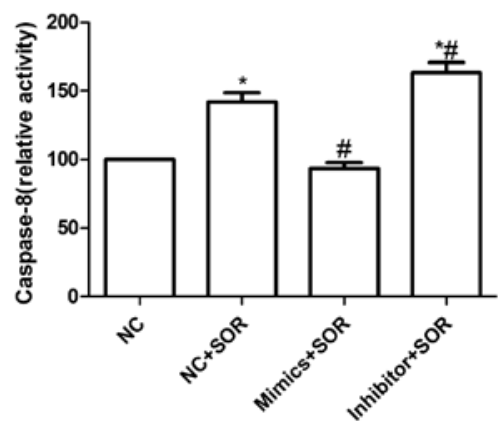

Figure 6. Effects on cellular proliferation and apoptosis of combined treatment with sorafenib (SOR) and miR-222 in HepG2 cells. HepG2 cells were transfected with miR-222 mimics or miR-222 inhibitor and corresponding negative control (NC) for $48 \mathrm{~h}$ and then further treated with indicated concentrations of sorafenib (10 $\mu \mathrm{M})$. After $72 \mathrm{~h}$, cell viability was determined by CCK8 assay (A), and apoptosis was analyzed by TUNEL (B), as well as caspase-3 (C) and caspase-8 (D) activity was determined by ELISA, respectively. ${ }^{*} \mathrm{P}<0.05$ versus NC (negative control); ${ }^{\#} \mathrm{P}<0.05$ versus NC+SOR. 


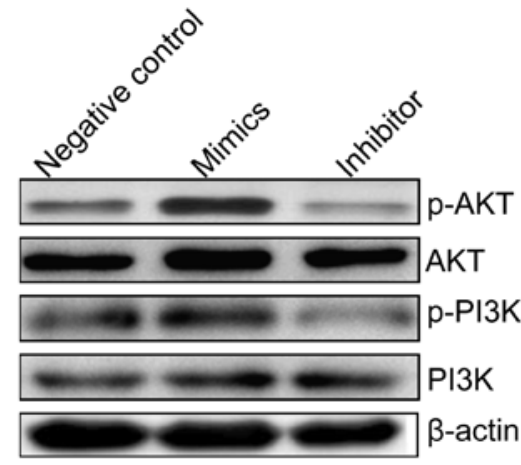

Figure 7. Effect of miR-222 on PI3K/AKT signaling pathway in HepG2 cells. Phosphorylation/activation pattern of PI3K and AKT were measured by western blotting $24 \mathrm{~h}$ after transfection with miR-222 mimics, miR-222 inhibitor and corresponding negative control in HepG2 cells. Blots were reprobed for $\beta$-actin to normalize each lane for protein content.

We also evaluated the effect of expression of miR-222 with the sorafenib on cellular apoptosis. The quantification of TUNEL-positive HepG2 cells showed that miR-222 mimic combination with sorafenib can decrease HepG2 cell apoptosis compared to sorafenib combination with negative control $(\mathrm{P}<0.05$, Fig. 6B), on the contrary, miR-222 inhibitor combination with sorafenib can induce cell apoptosis compared to sorafenib combination with negative control $(\mathrm{P}<0.05$, Fig. 6B). In addition, we evaluated the effect of expression of miR-222 with sorafenib on caspase- 3 and -8 activity. Compared to sorafenib combination with negative control treatment, miR-222 mimic combination with sorafenib could decrease caspase- 3 and -8 activity $(\mathrm{P}<0.05$, Fig. $6 \mathrm{C}$ and $\mathrm{D})$, whereas, miR-222 inhibitor combination with sorafenib increased caspase -3 and -8 activity ( $P<0.05$, Fig. 6 C and $D)$. These results suggested that miR-222 might confer sorafenib resistance in HepG2 cells.

miR-222 activates the PI3K/AKT signaling pathway. To clarify the molecular mechanisms involved in miR-222 effect on cell proliferation and survival of HCC cells, we focused on the effects of miR-222 expression on the PI3K/AKT pathway, which participate in the main intracellular signaling required for cell proliferation and drug-resistance in HCC cells. Measurements of the phosphorylation/activation pattern of PI3K and AKT were performed using western blotting. It was found that overexpression of miR-222 by transfection with miR-222 mimic resulted in a marked increase of phosphorylated PI3K and AKT relative to cells transfected with negative control, one the contrary, down-regulation of miR-222 by transfection miR-222 inhibitor resulted in a marked decrease of phosphorylated PI3K and AKT relative to cells transfected with negative control, without altering the total protein levels of PI3K and AKT in each group (Fig. 7). These results indicate that miR-222 affects cell proliferation, enhance the resistance of HCC cells to sorafenib, to some extent, by regulation of the PI3K/AKT signaling pathway.

\section{Discussion}

Several studies have shown that miR-222 are upregulated in various cancers and are considered oncogenes (19-23).
Specially, Wong et al found that miR-222 expression was increased in a larger series of primary HCC tumors compared with non-tumor livers, and a strong relationship was established between the high expression of miR-222 with tumor progression and patient survival (23). Consistent with these studies, our results showed that miR-222 expression level was elevated in most HCC tumor tissue compared to non-tumor tissue, and its expression level correlated with key pathological characteristics including tumor differentiation, stage, metastasis, and tumor size. No correlations occurred between miR-222 levels and patient age, gender and Child-Pugh grade. In addition, our results also showed that high miR-222 expression correlated with shorter overall survival and the miR-222 expression status in tumors can predict HCC survival. These results provide evidence that miR-222 may be a diagnosticmarker in HCC.

Human hepatocellular carcinoma is the leading cause of cancer death in both men and women worldwide. Recurrent disease is one of the most serious challenges for managing patients with HCC (29). Despite hepatic resection is a wellaccepted therapy for early-stage HCC, the prognosis of many patients is poor due to frequent intrahepatic metastasis and tumor recurrence (30). One of the most important factors that affect survival rate is resistance to therapeutic drugs. Therefore, development of effective therapeutic approaches is necessary for the management of HCC. Sorafenib is a recent FDA-approved anticancer drug that improved the overall survival of HCC patients (31). In recent years sorafenib has been used to treat advanced HCCs improving the overall survival of HCC patients from 7.9 to 10.7 months and it is the sole systemic drug that is proved to be effective in treatment for HCC $(31,32)$. However, treatment outcomes are still poor due to unfavorable pharmacokinetics, low tumor accumulation and other adverse effects (33). For this reason, it is necessary to decrease the toxic side effects of sorafenib and increase it sensitive to HCC cells. Recently, the correlation between miRNA expression and chemoresistance or sensitivity has also aroused widespread concern in several types of cancers, including HCC. Importantly, several in vitro data suggest that some miRs may sensitize the effects of sorafenib in HCC cells. For example, an miR-122 mimetic alone or in combination with sorafenib reduced the tumorigenic properties of HCC cells and may therefore be a promising therapeutic regimen for liver cancer (34). Yang et al found that miR-34a can induce apoptosis and modulate the sensitivity of HCC cells to sorafenib, at least in part, through regulating the $\mathrm{Bcl}-2$ expression (35). Similar to these studies, our result showed that miR-222 inhibitor combined with sorafenib in HCC cells could significantly reduce cell proliferation, induce cell apoptosis, increased the activity of caspase- 3 and -8 compared to sorafenib. These results might imply that miR-222 confers sorafenib resistance in HCC cells.

miR-221 and miR-222 share the same seed sequence, which are short, evolutionarily conserved regions through which miRNAs bind their target sites in mRNA 3'-UTRs, indicating an important role in coordinated regulation and function. Increasing evidence suggests miR-221 and miR-222 play important roles in cancer development, progression, metastasis and may be effective biomarkers for cancer prognosis. 
Importantly, studies have found that miRNAs affect a series of biological processes through complementary binding to one or several target genes. For instance, Galardi et al showed that in pancreatic cells, p2 $7^{\mathrm{Kip} 1}$ and miR-221/222 expression levels inversely correlated and demonstrated that miR221/222 overexpression had important consequences on the proliferation rate and the cell cycle phase distribution (36). Garofalo and his collaborators showed miR-221/222, targeting PTEN and TIMP3 tumor suppressors (25). Yang et al suggested that SIRT1 plays a suppressive role against the tumor promoting action of miR-221 and miR-222 (37). In addition, ADAM17, ARHI and HECTD2 were identified as the target genes of miR-221 and miR-222 $(22,38,39)$. These findings suggest that miR-221 and miR-222 are a therapeutic target. Therefore, we selected miR-222 as study target to observe its roles in HCC development and procession. It was found that downregulation of miR-222 by miR-222 inhibitor expression inhibited cell proliferation and migration and increased apoptosis in HepG2 cells. Moreover, effective transfection of miR-222 inhibitor resulted in a higher percentage of cells in G0/G1 phases and a lower percentage of cells in $\mathrm{S}$ phase. These findings indicate that inhibition of miR-222 expression exerts important biological effects on cell proliferation, apoptosis, cell migration, cell cycle distribution, and cell transition in HepG2.

Phosphoinositide 3-kinase/AKT signaling is one of the important oncogenic pathways and is frequently activated during liver tumorigenesis, and play an important role in HCC development and procession $(40,41)$. In vitro functional studies have also demonstrated that the AKT pathway can act as a critical mediator in the control of HCC cell invasion and motility $(42,43)$. Wong et al showed that miR-222 overexpression is common in HCC and could confer metastatic potential in HCC cells, possibly through activating AKT signaling. Importantly, it has been shown that activation of the PI3K/Akt signaling pathway can confer resistance to sorafenib in HCC cells (44). Therefore, in the present study, we focused on the effects of miR-222 expression on the PI3K/AKT pathway. Our result showed that overexpression miR-222 resulted in a marked increase of phosphorylated PI3K and AKT, whereas, down-expression miR-222 resulted in a marked decrease of phosphorylated PI3K and AKT, without altering the total protein levels of PI3K and AKT in each group. These findings suggest that PI3K/AKT signaling is the major pathway influenced by miR-222, and that miR-222 confer resistance to sorafenib in HCC cells, at least in part, via PI3K/AKT pathway.

In conclusion, the findings reported here present evidence that miR-222 can promote cell proliferation, migration and invasion, and decrease cell apoptosis, as well as enhance the resistance of HCC cells to sorafenib, at least in part, through activating PI3K/AKT signaling pathway. Our data suggest that manipulating miRNA expression may be useful for future development of chemo-sensitizing strategies and treatment for HCC.

\section{Acknowledgements}

The authors gratefully acknowledge the financial support provided by The Development of Science and Technology Plan Projects of Jilin (no. 209Z0198).

\section{References}

1. Jemal A, Bray F, Center MM, Ferlay J, Ward E and Forman D: Global cancer statistics. CA Cancer J Clin 61: 69-90, 2011.

2. Welzel TM, Graubard BI, Zeuzem S, El-Serag HB, Davila JA and McGlynn KA: Metabolic syndrome increases the risk of primary liver cancer in the United States: a study in the SEER-Medicare database. Hepatology 54: 463-471, 2011.

3. Thorgeirsson SS and Grisham JW: Molecular pathogenesis of human hepatocellular carcinoma. Nat Genet 31: 339-346, 2002.

4. Newell P, Villanueva A and Llovet JM: Molecular targeted therapies in hepatocellular carcinoma: from pre-clinical models to clinical trials. J Hepatol 49: 1-5, 2008.

5. Ma P and Mumper RJ: Anthracycline nano-delivery systems to overcome multiple drug resistance: a comprehensive review. Nano Today 8: 313-331, 2013.

6. Vanderlaag K, Wang W, Fayadat-Dilman L, et al: Regenerating islet-derived family member, 4 modulates multiple receptor tyrosine kinases and mediators of drug resistance in cancer. Int $\mathbf{J}$ Cancer 130: 1251-1263, 2012

7. Baguley BC: Multiple drug resistance mechanisms in cancer. Mol Biotechnol 46: 308-316, 2010

8. Schneider E and Cowan KH: Multiple drug resistance in cancer therapy. Med J Aust 160: 371-373, 1994.

9. Pillai RS, Bhattacharyya SN, Artus CG, et al: Inhibition of translational initiation by Let-7 MicroRNA in human cells. Science 309: 1573-1576, 2005.

10. Anglicheau D, Muthukumar T and Suthanthiran M: MicroRNAs: small RNAs with big effects. Transplantation 90: 105-112, 2010

11. Calin GA, Dumitru CD, Shimizu M, et al: Frequent deletions and down-regulation of micro- RNA genes miR15 and miR16 at $13 q 14$ in chronic lymphocytic leukemia. Proc Natl Acad Sci USA 99: 15524-15529, 2002.

12. Takamizawa J, Konishi $\mathrm{H}$, Yanagisawa $\mathrm{K}$, et al: Reduced expression of the let-7 microRNAs in human lung cancers in association with shortened postoperative survival. Cancer Res 64: 3753-3756, 2004

13. Meng F, Henson R, Lang M, et al: Involvement of human micro-RNA in growth and response to chemotherapy in human cholangiocarcinoma cell lines. Gastroenterology 130: 2113-2129, 2006.

14. Woods K, Thomson JM and Hammond SM: Direct regulation of an oncogenic micro-RNA cluster by E2F transcription factors. J Biol Chem 282: 2130-2134, 2007.

15. Wang $\mathrm{H}, \mathrm{Xu} \mathrm{C}$, Kong $\mathrm{X}$, et al: Trail resistance induces epithelial-mesenchymal transition and enhances invasiveness by suppressing PTEN via miR-221 in breast cancer. PLoS One 9: e99067, 2014

16. Kutanzi KR, Yurchenko OV, Beland FA, Checkhun VF and Pogribny IP: MicroRNA-mediated drug resistance in breast cancer. Clin Epigenetics 2: 171-185, 2011.

17. Stinson S, Lackner MR, Adai AT, et al: miR-221/222 targeting of trichorhinophalangeal 1 (TRPS1) promotes epithelial-to-mesenchymal transition in breast cancer. Sci Signal 4: pt5, 2011.

18. Yang CJ, Shen WG,Liu CJ, et al: $\mathrm{miR}-221$ and miR-222 expression increased the growth and tumorigenesis of oral carcinoma cells. J Oral Pathol Med 40: 560-566, 2011.

19. Lu Y, Roy S, Nuovo G, et al: Anti-microRNA-222 (anti-miR-222) and -181B suppress growth of tamoxifen-resistant xenografts in mouse by targeting TIMP3 protein and modulating mitogenic signal. J Biol Chem 286: 42292-42302, 2011.

20. Miller TE, Ghoshal K, Ramaswamy B, et al: MicroRNA-221/222 confers tamoxifen resistance in breast cancer by targeting p $27^{\mathrm{Kipl}}$. J Biol Chem 283: 29897-29903, 2008.

21. Zhang CZ, Zhang JX, Zhang AL, et al: MiR-221 and miR-222 target PUMA to induce cell survival in glioblastoma. Mol Cancer 9: 229, 2010.

22. Xu K, Liang X, Shen K, et al: MiR-222 modulates multidrug resistance in human colorectal carcinoma by down-regulating ADAM-17. Exp Cell Res 318: 2168-2177, 2012.

23. Wong QW, Ching AK, Chan AW, et al: MiR-222 overexpression confers cell migratory advantages in hepatocellular carcinoma through enhancing AKT signaling. Clin Cancer Res 16: 867-875, 2010.

24. Zhong S, Li W, Chen Z, Xu J and Zhao J: MiR-222 and miR-29a contribute to the drug-resistance of breast cancer cells. Gene 531: 8-14, 2013.

25. Garofalo M, Di Leva G, Romano G, et al: miR-221\&222 regulate TRAIL resistance and enhance tumorigenicity through PTEN and TIMP3 downregulation. Cancer Cell 16: 498-509, 2009. 
26. Wittekind C: Pitfalls in the classification of liver tumors. Pathologe 27: 289-293, 2006 (In German).

27. Tio TL: The TNM staging system. Gastrointest Endosc 43: S19-S24, 1996

28. Gao Q, Qiu SJ, Fan J, et al: Intratumor balance of regulatory and cytotoxic $\mathrm{T}$ cells is associated with prognosis of hepatocellular carcinoma after resection. J Clin Oncol 25: 2586-2593, 2007.

29. El-Serag HB: Hepatocellular carcinoma. N Engl J Med 365: 1118-1127, 2011.

30. Bruix J, Sherman M and American Association for the Study of Liver D: Management of hepatocellular carcinoma: an update. Hepatology 53: 1020-1022, 2011

31. Llovet JM, Ricci S, Mazzaferro V, et al: Sorafenib in advanced hepatocellular carcinoma. N Engl J Med 359: 378-390, 2008.

32. Liu L, Cao Y, Chen C, et al: Sorafenib blocks the RAF/MEK/ ERK pathway, inhibits tumor angiogenesis, and induces tumor cell apoptosis in hepatocellular carcinoma model PLC/PRF/5. Cancer Res 66: 11851-11858, 2006.

33. Roy M, Luo YH, Ye M and Liu J: Nonsmall cell lung cancer therapy: insight into multitargeted small-molecule growth factor receptor inhibitors. Biomed Res Int 2013: 964743, 2013.

34. Bai S, Nasser MW, Wang B, et al: MicroRNA-122 inhibits tumorigenic properties of hepatocellular carcinoma cells and sensitizes these cells to sorafenib. J Biol Chem 284: 32015-32027, 2009.

35. Yang F, Li QJ, Gong ZB, et al: MicroRNA-34a targets Bcl-2 and sensitizes human hepatocellular carcinoma cells to sorafenib treatment. Technol Cancer Res Treat 13: 77-86, 2014.

36. Galardi S, Mercatelli N, Giorda E, et al: miR-221 and miR-222 expression affects the proliferation potential of human prostate carcinoma cell lines by targeting p27 ${ }^{\mathrm{Kipl}}$. J Biol Chem 282: 23716-23724, 2007.
37. Yang X, Yang Y, Gan R, et al: Down-regulation of miR-221 and miR-222 restrain prostate cancer cell proliferation and migration that is partly mediated by activation of SIRT1. PLoS One 9: e98833, 2014.

38. Chen Y, Zaman MS, Deng G, et al: MicroRNAs 221/222 and genistein-mediated regulation of ARHI tumor suppressor gene in prostate cancer. Cancer Prev Res 4: 76-86, 2011.

39. Sun T, Wang X, He HH, et al: MiR-221 promotes the development of androgen independence in prostate cancer cells via downregulation of HECTD2 and RAB1A. Oncogene 33: 27902800,2014

40. Li W, Tan D, Zhang Z, Liang JJ and Brown RE: Activation of Akt-mTOR-p70S6K pathway in angiogenesis in hepatocellular carcinoma. Oncol Rep 20: 713-719, 2008

41. Chen JS, Wang Q, Fu XH, et al: Involvement of PI3K/PTEN/ $\mathrm{AKT} / \mathrm{mTOR}$ pathway in invasion and metastasis in hepatocellular carcinoma: association with MMP-9. Hepatol Res 39: 177-186, 2009.

42. Krasilnikov M, Ivanov VN, Dong J and Ronai Z: ERK and PI3K negatively regulate STAT-transcriptional activities in human melanoma cells: implications towards sensitization to apoptosis. Oncogene 22: 4092-4101, 2003

43. Saxena NK, Sharma D, Ding X, et al: Concomitant activation of the JAK/STAT, PI3K/AKT, and ERK signaling is involved in leptin-mediated promotion of invasion and migration of hepatocellular carcinoma cells. Cancer Res 67: 2497-2507, 2007.

44. Chen KF, Chen HL, Tai WT, et al: Activation of phosphatidylinositol 3-kinase/Akt signaling pathway mediates acquired resistance to sorafenib in hepatocellular carcinoma cells. JPharmacol Exp Ther 337: 155-161, 2011. 